\title{
Yves Thoret (1938-2020)
}

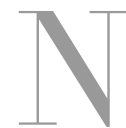

é en 1938, son père était médecin, Yves Thoret fut chef de service au CHS de Bécheville, les Mureaux, tout en enseignant en psychopathologie à Nanterre, où je l'ai mieux connu. Il avait soutenu sa thèse en 1968, sous la direction de P. Pichot, qui portait sur l'automatisme mentale. C'était à Nanterre, un enseignant devenant au fil des années de plus en plus atypique par sa culture et son ascendant et en raison de la volonté qu'il montrait et assumait de ne pas robotiser les étudiants. Avec sa disparition, Nanterre vit se marquer la fin de sa période historique en psychologie clinique et pathologique, ce fut une période de grand renom, Yves voisinait alors avec R. Dorey, D. Anzieu, B. Gibello, entre autres enseignants d'envergure.

Peu après son départ de Nanterre, il m’invita à rejoindre une de ses maison, à laquelle il portait un attachement sérieux : la revue l'Évolution Psychiatrique, je fis partie de la rédaction alors que Christophe Chaperot avait succédé à Yves qui dirigea cette la revue de 1991 à 2003.

Personnalité de belle trempe, et habile négociateur, il avait protégé cette revue, lui permettant les indexations nécessaires et contribuant en cela à en accroitre le renom national et international. Je partageais avec lui cet intérêt pour des échanges avec des collègues de l'étranger et nous avons pris en charge la rubrique correspondants internationaux.

La personnalité de Yves était celle d'un caractère entier, généreux et enthousiaste. L'humeur souvent bouillonante se tempérait vite par des traits d'humour et d'ironie qui ravissaient, stimulaient ou bousculaient parfois.

Infatigabe il fut aussi directeur scientifique à la célèbre Encyclopédie Médico-chirurgicale. Yves Thoret, passionné de théâtre, fit sa thèse avec Didier Anzieu (doctorat d'État, 1988 ) sur la théâtralité. Il en fit la matière d'un livre, toujours actuel et paru chez Dunod en 1993 : La théatralité, étude freudienne. Sur ses « autres scènes " que furent les voyages et le théâtre il était intarissable, pétillant de culture. Il sut conserver et exalter son amour du théâtre pour repenser la question de la représentation, de l'illusion et de l'espace intermédiaire.

Le fil de l'amitié, vite noué entre nous, est resté solide tout au long de nos années commnunes à l'Évolution qu'il ne quitta qu'en 2020.

Auteur, directeur de publications scientifiques, amoureux de l'art, toujours à l'affut de ce qui est vrai et neuf, Yves a marqué la psychiatrie, l'enseignement et l'édition.

Olivier Douville 
Cette année décidément n’en finit pas de nous apporter des nouvelles affligeantes. Cette fois, en tournant la page des annonces du “ monde » c'est la mort d'Yves Thoret qui me saute au visage. Yves, bon sang, non pas toi. Mais pourtant si. Pas d'erreur. La camarde a décidé de ne rien nous épargner. Yves c'était toujours le sourire moqueur de quelqu'un qu'on ne va pas baratiner avec n'importe quelle sottise. Yves c'était la “Commission Psychiatrie» en 68. Yves c'était l'amitié dans sa simplicité. Toto, c'était l'amour du théâtre aussi. Les théâtres sont fermés, ils sont en berne avant même d'apprendre la nouvelle. Ils ont su sans doute avant nous.

Décidément j'en ai plus qu'assez de cette année 2020. Vivement qu'on tourne enfin la page. Yves, tu vas trop nous manquer.

Déjà que ce n'était pas gai mais là, je trouve qu'il y a vraiment de l'abus.

Laurent Le Vaguerèse 